OPEN ACCESS

Edited by:

Miloslav Pekar

Brno University of Technology,

Czechia

Reviewed by:

Dave John Adams,

University of Glasgow,

United Kingdom

Myriam Haydee Aguirre,

University of Zaragoza, Spain

*Correspondence:

Viness Pillay

Viness.Pillay@wits.ac.za

Specialty section:

This article was submitted to Colloidal Materials and Interfaces,

a section of the journal

Frontiers in Materials

Received: 23 October 2019

Accepted: 13 March 2020

Published: 30 April 2020

Citation:

Ramiah P, du Toit LC,

Choonara YE, Kondiah PPD and

Pillay V (2020) Hydrogel-Based

Bioinks for 3D Bioprinting in Tissue

Regeneration. Front. Mater. 7:76.

doi: 10.3389/fmats.2020.00076

\section{Hydrogel-Based Bioinks for 3D Bioprinting in Tissue Regeneration}

\author{
Previn Ramiah, Lisa C. du Toit, Yahya E. Choonara, Pierre P. D. Kondiah and \\ Viness Pillay* \\ Wits Advanced Drug Delivery Platform Research Unit, Department of Pharmacy and Pharmacology, Faculty of Health \\ Sciences, University of the Witwatersrand, Johannesburg, Johannesburg, South Africa
}

Three-dimensional (3D) printing of biomaterials provides an interesting alternative for the production of allograft tissues and organs to circumvent the incidences of donor scarcity and organ shortages. With the current deficit of readily available and viable organs for transplantation, the medical sector is faced with an increasing demand for organs and the shortfall in supply. Over the past decades, tissue engineering (TE) and regenerative medicine continue to provide alternative strategies for artificial tissues and organs. Current research shows that employing hydrogels as a cell-laden bioinks for the fabrication of 3D tissue constructs enables a lack of immunogenicity, since the hydrogelbased bioink is patient-specific and derived from biopolymers that demonstrate excellent biocompatibility and biodegradability, decreased organ rejection, increased organ viability, and enhanced the supply in accordance to the demand. While sufficient evidence directs researchers to conclude the safe and efficacious process of seeding cells, biomolecules, and biomaterials using 3D bioprinting, there are multiple limitations, which requires significant attention, such as cost, volumetric bioprinting, integrity, and strength of biomaterials, as well as multicellular and multimaterial bioprinting. In this review, the focus is on the applications of hydrogels as bioinks employed in 3D bioprinting and, where applicable, considerations of note and challenges encountered. This review proposes to highlight not only the progress forged in this area, but also the limitations of hydrogel-based bioink investigations to date and the need for further multidisciplinary investigation and progression to the stage of clinical testing of human-scale tissue constructs.

Keywords: hydrogel, 3D bioprinting, bioink, volumetric printing, tissue regeneration, 4D printing

\section{INTRODUCTION}

Three-dimensional (3D) bioprinting (biomimicry) refers to the process of producing cell-laden models into functional tissue and organs for transplantation and drug testing. The recent interest and popularity in 3D bioprinting confirms the potential for 3D bioprinting technology to decrease crippling burden of fabrication processes in reconstructive or regenerative medicine (Thomas, 2016; Vijayavenkataraman et al., 2018). 3D bioprinting provides tissue engineering (TE) researchers with bioinks (based on biomaterials) to 3D print biologically relevant structures. Many of these biomaterials have demonstrated an increase in strength and durability compared with the original tissue or organ. Furthermore, bioinks are often based on hydrogel frameworks. 
Alginate, for example, commonly used in 3D bioprinting, possesses low toxicity and increased biocompatibility and is cost-effective when compared to other biomaterials. This research field and future developments will extend from producing hydrogel-based bioinks with enhanced physicochemical attributes to incorporating microchannels to allow for effective nutrient diffusion for resident bioprinted cells (Vijayavenkataraman et al., 2018).

Three-dimensional bioprinting confers several advantages over conventional tissue seeding for TE. It allows for the precise placement of cells, biomolecules, and biomaterials in a spatially predefined 3D position. 3D bioprinting encompasses a variety of methods such as selective laser sintering, extrusion, and inkjet bioprinting, which reduces the limitations of conventional TE, upon the incorporation of a computer-aided design (CAD), which gives rise to the precise placement of cells, within the confined 3D structure, as illustrated in Figure 1 (Mironov et al., 2006; Billiet et al., 2012; Derby, 2012; Seol et al., 2014).

Three-dimensional bioprinting can be used to produce biomimetic structures based on a computed tomographic image obtained from a patient's damaged or injured body organ and shifting these data to a CAD, which directs the production of a patient specific structure. This process is essentially the conversion of a scanned 2D image into a CAD as illustrated in Figure 2 (Billiet et al., 2014).

While sufficient evidence directs researchers to conclude the safe and efficacious process of seeding cells, biomolecules, and biomaterials using 3D bioprinting, there are multiple limitations, which requires significant attention, such as cost, volumetric bioprinting, integrity, and strength of biomaterials, as well as multicellular and multimaterial bioprinting. In this review, the focus is on the applications, considerations, and limitations of hydrogels as bioinks employed in 3D bioprinting.

\section{APPROACHES TO 3D BIOPRINTING HYDROGELS FOR TISSUE REGENERATION}

The primary approach of $3 \mathrm{D}$ bioprinting is to provide homogenous cell seeding and precise placement of cells to generate complex multifunctional structures. The placement of cells involves implanting cells onto a biodegradable scaffold (including chemical growth hormones), which are required for the growth and development of cells for TE (Blaeser et al., 2013; Zhang and Zhang, 2015).

The conventional approach to $\mathrm{TE}$ requires incorporating isolated cells onto a porous scaffold, which is then followed by in vivo implantation (Pereira and Bártolo, 2015). Polyglycolic acid (PGA) has been used as a polymer of choice, owing to its thermoplastic and biodegradability (Langer and Vacanti, 1993). This approach to TE bears multiple limitations that include the following:

- Organs and tissues are multifunctional structures, comprising an array of different cellular species that require specific positioning for effective functioning, which posed a critical technical problem as conventional $\mathrm{TE}$ lacked the ability to specifically position cells or tissues (Boland et al., 2003).

- Polyglycolic acid, as the polymer of choice, lacks contractibility and limits this application for rheumatology (Boland et al., 2003).

- Cellular placement, development, and attachment require a time scale from weeks to months. Cellular placement lacks uniformity (Boland et al., 2003).

- Conventional volumetric TE is limited by the absence of vascular structures (Boland et al., 2003).

At present, these limitations can be addressed by using $3 \mathrm{D}$ bioprinting as a technique for TE. Computer-aided processes are used to develop a 3D organization of living cells within a temporary biodegradable scaffold. This allows for rapid volumetric printing and production of complex multicellular organs. 3D bioprinting also allows for the simultaneous dispensing of biomaterials and cells, resulting in seeding efficiency and the prevention of non-homogenous cell distribution because of postfabrication seeding (Guillemot et al., 2010a; Pereira and Bártolo, 2015; De Maria et al., 2017).

\section{Three-Dimensional Bioprinting Techniques for Fabricating Cell-Laden Hydrogel-Based Bioinks}

Table 1 illustrates the limitations in TE that can be circumvented by the use of $3 \mathrm{D}$ bioprinting as a bioengineering tool for the fabrication of multifunctional cell-laden hydrogel structures for tissue regeneration (Mandrycky et al., 2016).

\section{Inkjet Bioprinting}

Inkjet bioprinting is the process of dispensing picoliter droplets of the bioink (consisting of cells and biofactors), on to a substratum, through a non-contact process (Lee et al., 2010). The advantages of employing inkjet bioprinting cell-laden hydrogels include increased printing speed, which can be attributed to the ability of printer nozzle to allow for parallel workflow; relatively high cell viability; and decreased acquisition and utilization costs (Zhang and Zhang, 2015). Inkjet bioprinting can be divided into two broad classes, based on the mechanism employed to generate the droplet for dispensing:

\section{Thermal inkjet bioprinting}

Encompasses the electrical heating of the print head, generating pressure pulses, and induces ejection of the droplet by inducing a bubble formation, which stimulates ejection of the droplet of $10-15 \mathrm{pL}$. The required temperature for this technique ranges between 200 and $300^{\circ} \mathrm{C}$ for a period of $2 \mu \mathrm{s}$. Because of the short heating duration, there is only a deviation of $4-10^{\circ} \mathrm{C}$ of the total system; thus, there is no significant damage to the biological substances (Cui et al., 2010).

\section{Piezoelectric inkjet bioprinting}

This technique requires the use of an actuator or piezoelectric crystal, which produces an auditory wave within the printer head upon an applied voltage. This wave breaks the liquid and induces dispensing of droplets at regular intervals (Tekin et al., 2008). 


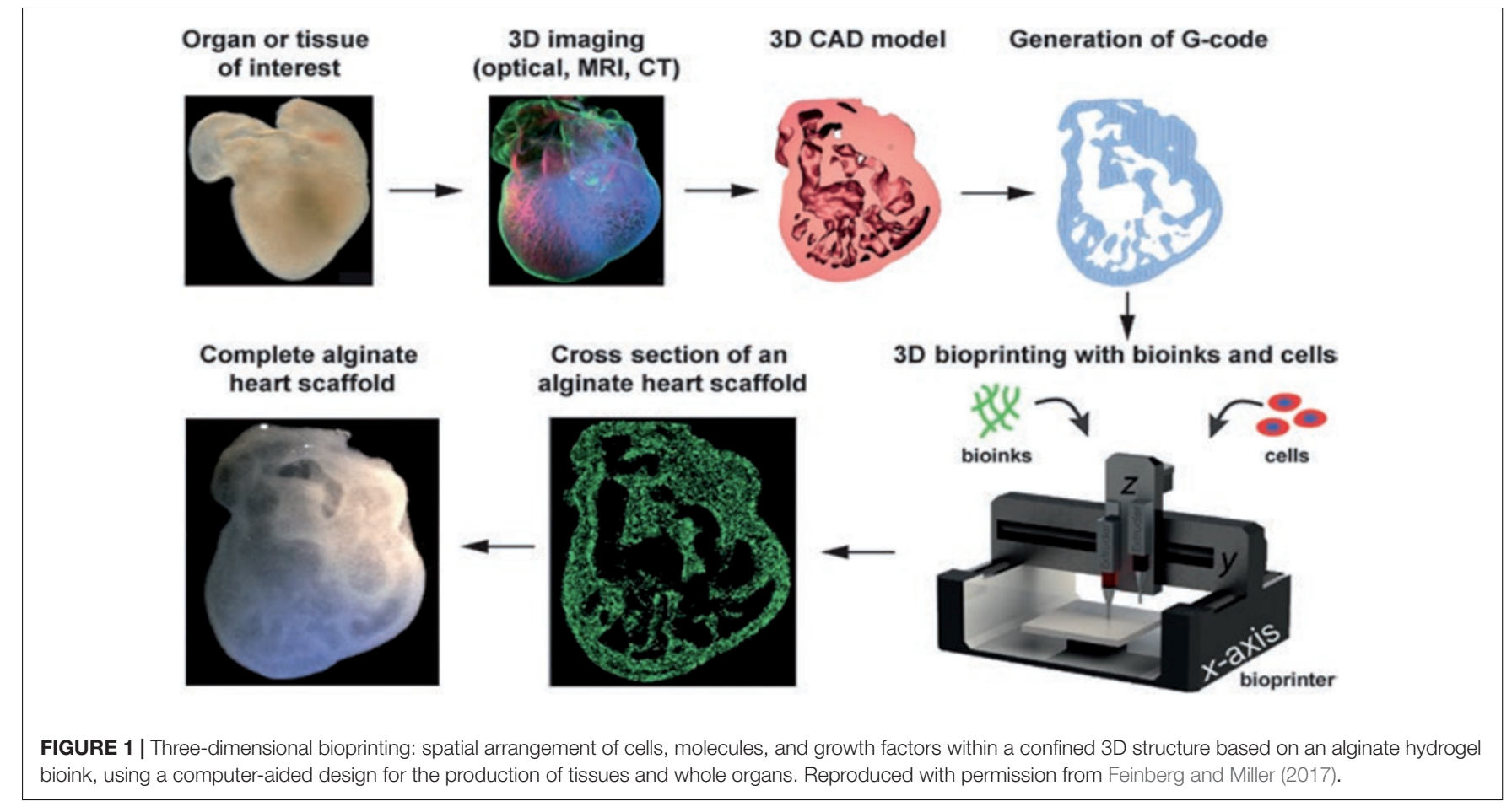

Auditory waves generated within the range of $15-25 \mathrm{kHz}$ should be avoided, as frequencies within this range result in cellular damage (Cui et al., 2012).

\section{Microextrusion Bioprinting}

Microextrusion 3D bioprinter comprises a stage, which can move along three orthogonal planes, XYZ; a temperature-controlled dispensing cartridge; a fiber-optic light-illumined deposition area; a humidifier; and a video camera (Zhang and Zhang, 2015; Feinberg and Miller, 2017). Microextrusion generates constant beads of biomaterial that is dispensed in two dimensions in accordance to a CAD, unlike inkjet bioprinting, which eject droplets. Each deposited layer serves as a foundation for a following layer, while the stage or the printer head moves along the $Z$-axis (Zhang and Zhang, 2015).

\section{Laser-Assisted Bioprinting}

Laser-assisted bioprinters (LABs) consist of the following components (Ringeisen et al., 2006):

- Pulsed laser source

- Ribbon from which the biological material is printed from

- Receiving platform.

The process of LAB encompasses a multilayer ribbon consisting of a support; the support is a transparent layer relative to the laser radiation wavelength, which is then coated with a transfer layer, commonly referred to as the bioink. The optical properties of the bioink or the wavelength of the laser directs whether that the addition of a laser-absorbing layer between the support and bioink will induce ejection (Guillemot et al., 2010b).

\section{HYDROGELS AS INNOVATIVE BIOINKS}

The selection of a bioink is as important as selecting the technique used for 3D bioprinting (Cui et al., 2017). Bioink selection for TE is critical as it should provide an array of biochemical and physical cues that promote cellular growth, development, and proliferation. These cues include chemokines, growth and adhesion factors, and mechanical and physical properties as exhibited by the extracellular matrix (ECM) of native cells (Griffith and Swartz, 2006). For the field of TE, bioinks aid as a biomimetic ECM that stimulates regeneration of tissue growth and proliferation (Cui et al., 2017).

\section{Considerations for the 3D Bioprinting Process of Hydrogels}

At present, "soft biomaterials" used for developing cell-laden structures and providing a conducive environment for cellular growth and development are often hydrogel based.

Hydrogels are defined as 3D networks comprising crosslinked hydrophilic polymer chains, distinguished by their high water content and array of biophysical properties. Hydrogels can be designed and printed into a variety of shapes, sizes, and forms to meet the final product requirements. The attractive properties of hydrogels are that it can be engineered to mimic the extracellular tissue microenvironment, enabling its medical application as biosensors, scaffolds for tissue regeneration, and drug delivery technology (Hoffman, 2012; Kamata et al., 2015). Hydrogels are attractive as cell carriers in TE, stem cell, and cancer research, owing to recent advances in $3 \mathrm{D}$ bioprinting. Over the past decade, hydrogels have received significant attention; thus, substantial progress has been made 


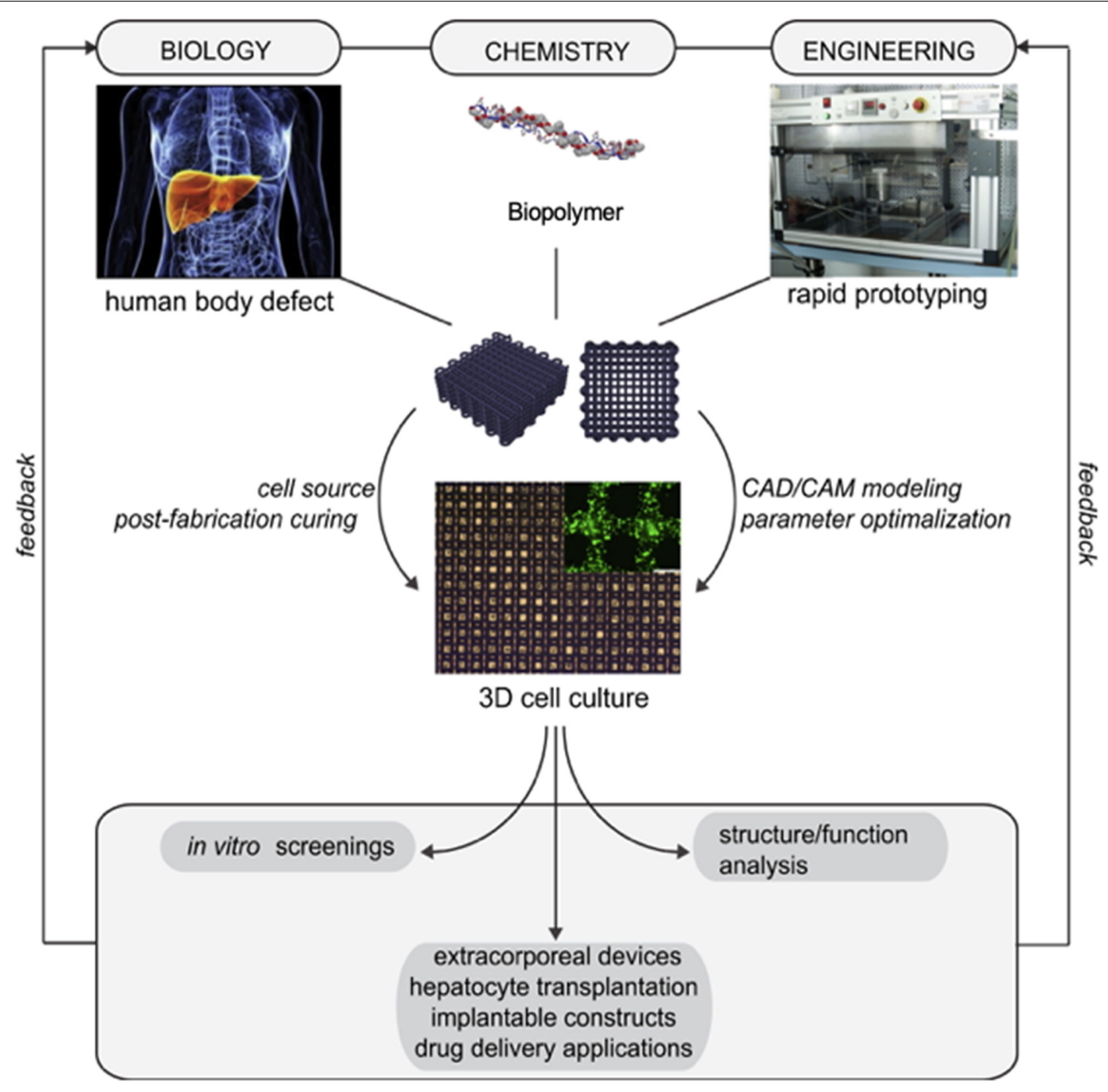

FIGURE 2 | Diagrammatic representation of the sequential process for the fabrication of patient specific structures. Adapted with permission from Billiet et al. (2014).

in the modification of the physicochemical properties through gelation, click chemistry, and incorporation of nanoparticles (Zhang and Khademhosseini, 2017).

Consideration must be given to the limitations of certain hydrogels that, upon implantation, can induce various side effects stimulated by polymerization residues, such as monomers, reaction initiators, and catalysts (Peppas and Khademhosseini, 2016). Residual methyl methacrylate monomer, generated during the polymerization of poly(methyl methacrylate), results in cellular damage and death, central nervous system damage, and skin and ocular irritation. The long-term release of these toxic substances is attributed to erosion and degradation of the polymer network over time (Gupta et al., 2012; Kamata et al., 2015). Solutions to overcome these limitations include application of a reversible-deactivation radical polymerization technique, which periodically attaches and detaches active molecules or residues, preventing the undesirable outcomes produced during the polymerization process (Kamata et al., 2015). This further rationalizes that application of a biopolymer in such instances would be more favorable.

\section{Preparation and Incorporation of Cells Within Hydrogel-Based Bioinks}

The species and specificity of cells needed for TE should greatly direct the choice of the $3 \mathrm{D}$ bioprinting approach applied. The nature of the required cell, such as age, source, and type, significantly impacts the success of the hydrogelbased bioink, and careful consideration should be applied for the $3 \mathrm{D}$ bioprinting process. The extraction procedure of the required cells is immensely critical, as the viability of cells during the preprinting phase is a requirement for the successful generation of a tissue-engineered structure. Temperature, oxygen, carbon dioxide, and $\mathrm{pH}$ levels are often ignored; however, the levels (Zhao et al., 2017a) of these substances should be monitored and controlled to ensure a 
TABLE 1 | Comparison of the different techniques employed for the fabrication of 3D bioprinted cell-laden hydrogel constructs for tissue regeneration.

\begin{tabular}{|c|c|c|c|}
\hline & Inkjet & Laser-assisted & Extrusion \\
\hline Cost & Low & High & Moderate \\
\hline Cell viability & $>85 \%$ & $>95 \%$ & $40-80 \%$ \\
\hline Print speed & Fast & Medium & Slow \\
\hline Supported viscosities & $3.5-12 \mathrm{mPa} / \mathrm{s}$ & $1-300 \mathrm{mPa} / \mathrm{s}$ & $30 \mathrm{mPa} / \mathrm{s}$ to above $6 \times 10^{7} \mathrm{mPa} / \mathrm{s}$ \\
\hline Resolution & High & High & Moderate \\
\hline Quality of vertical structure & Poor & Fair & Good \\
\hline Cell density & Low $<10^{6}$ cells $/ \mathrm{mL}$ & Medium $<10^{8}$ cells $/ \mathrm{mL}$ & High (cell spheroids) \\
\hline \multirow[t]{3}{*}{ Representative hydrogel materials for bioinks } & - Alginate & - Collagen & - Alginate \\
\hline & - PEGDMA & - Matrigel & - GelMA \\
\hline & - Collagen & & - Collagen \\
\hline \multirow[t]{8}{*}{ Reported applications in tissue engineering } & - Blood vessel & - blood vessel & - Blood vessel \\
\hline & - Bone & - bone & - Bone \\
\hline & - Cartilage & - skin & - Cartilage \\
\hline & - Neuron & - adipose & - Neuron \\
\hline & & & - Muscle \\
\hline & & & - Tumor \\
\hline & & & - Controlled release of biomacromolecules \\
\hline & & & - Organ-on-a-chip \\
\hline
\end{tabular}

Reproduced with permission from Mandrycky et al. (2016).

successful generation of a 3D tissue-engineered structure. After the successful extraction of the required cells, the cells should be gently mixed, avoiding the production of air bubbles and turbulent flow, to be incorporated into a bioink or its precursor (Rutz et al., 2017).

\section{The Effect of Cells on Hydrogel-Based Bioinks}

Three-dimensional bioprinting provides the platform for the production of an array of versatile cell-laded hydrogels as tissue constructs an in vitro pathological tissue models for $\mathrm{TE}$, screening applications, and drug testing ( $\mathrm{Xu}$ et al., 2011; Yao et al., 2012; Marchioli et al., 2015). Incorporation of cells into the hydrogel-based bioink can result in an alteration in the mechanical and rheological properties of the hydrogel-based bioink. These alterations would ultimately depend on the hydrogel structure. For example, in physically crosslinked gel-phase bioinks, including cells in the ink lowered the crosslinking degree and viscosity of the liquid phase and ultimately decreased the overall mechanical properties (Rutz et al., 2017). However, the success of the production of long-term stable structures with a concurrent increase in cell survival rate is significantly limited and dependent on the viscoelasticity of the hydrogel. An increase in the gelling or viscoelastic behavior of the cell-laden hydrogel is attributed to the most considerable causes of cellular death and injury (Chang et al., 2008). Currently, there are few studies that assessed the nature and extent of cells on the rheological behavior of a hydrogel-based bioink, because most researchers focus on the physicochemical properties of a cell-barren hydrogel. For physically crosslinked hydrogels, an increasing cell density often corresponds to a decrease in the crosslinking ability, viscosity, and final mechanical strength (Billiet et al., 2014).
Zhao et al. (2017a) demonstrated that incorporation of cells of regularly used density, $10^{6}$ cells $/ \mathrm{mL}$, exhibits a limited influence on the gelation temperature of the hydrogel but decreases the rheological properties of the hydrogel. This study explained this observation as the ability of incorporated cells to disturb the hydrogel network (Zhao et al., 2017a). Billiet et al. (2014) demonstrated the viscosity of a gelatin-methacrylamide hydrogel for the encapsulation of a hepatocarcinoma cell line was inversely proportional to the cell density. This study also demonstrated that an increasing cell density significantly contributed to the decrease in the moduli of thermally responsive gelatin-methacrylamide hydrogel. However, a decrease in the gelation temperature resulted in the compensation of the decreased mechanical strength attributed from the inclusion of cells (Billiet et al., 2014).

\section{Effect of Cell Survival on Hydrogel-Based Bioinks}

Zhao et al. (2015) demonstrated the effect of the concentration and composition of a gelatin-based hydrogel to determine the hydrogel's rheological effect on cell survival. This study demonstrated the inverse relationship between the cell survival rate and an increase in the viscoelastic nature of the hydrogel, which was attributed from an increase in the bioink concentration, an increase in the holding time, and a decrease in the holding temperature. This study also demonstrated that different process parameters that result in similar rheological behavior exhibit similar cell survival behavior (Zhao et al., 2015).

\section{Application of Hydrogel-Based Bioinks}

Table 2 illustrates the recent advances accomplished in the fabrication of 3D bioprinted cell-laden hydrogels for TE.

Sithole et al. (2018) demonstrated that sodium alginate was a polymer of choice, which is noted to have a good 
TABLE 2 | Applications of hydrogel-based bioinks for the fabrication of 3D bioprinted tissue constructs.

\begin{tabular}{|c|c|c|c|c|}
\hline Fabricated tissue & 3D bioprinting technique & Cellular material & Hydrogel polymer & References \\
\hline \multirow[t]{2}{*}{ Skin } & Ink jet-based bioprinting & - Fibroblasts & - Collagen & Lee et al., 2009 \\
\hline & & - Keratinocytes & & \\
\hline \multirow[t]{4}{*}{ Musculoskeletal tissue } & Extrusion-based bioprinting & - Endothelial progenitor cells & - Agarose & Fedorovich et al., 2008 \\
\hline & & - Bone marrow stromal cells & - Alginate & \\
\hline & & & - Hydroxyapatite & \\
\hline & & & - Polycaprolactone & \\
\hline Cardiac tissue & Extrusion-based bioprinting & - Human cardiac-derived cardiomyocyte progenitor cells & - Alginate & Gaetani et al., 2012 \\
\hline \multirow[t]{2}{*}{ Heart valve } & Extrusion-based bioprinting & - Aortic root sinus smooth muscle cells & - Alginate & Duan et al., 2013 \\
\hline & & - Aortic valve leaflet interstitial cells & - Gelatin & \\
\hline Neuronal tissue & Ink jet-based bioprinting & - Neural stem cell & - Polyacrylamide & Ilkhanizadeh et al., 2007 \\
\hline
\end{tabular}

biocompatibility, low toxicity, and stronger structural integrity compared to the original biological structure. The pore size of the polymer complex provides channels for effective nutrient and waste material exchange (an ideal environment for developing cells) (Sithole et al., 2018).

Degradation evaluations were carried out yielding Alg-PEI/Si with $50 \%$ initial degradation within the first $24 \mathrm{~h}$ but maintained the $3 \mathrm{D}$ structure until day 28 . This critical degradation parameter could be reduced by the addition of an alkaline inorganic filler, which reduces the acidic autocatalytic degradation of polymers (Stevens, 2008).

$\mathrm{Wu}$ et al. (2017) described the process of developing a TE scaffold for $3 \mathrm{D}$ bioprinting a liver-mimetic construct with an alginate/cellulose nanocrystal (CNC) hybrid bioink. The use of alginate has several advantages when incorporated as a hydrogel for TE, such as biocompatibility and easy processability. Under exposure of divalent cations such as $\mathrm{Ca}^{2+}$, alginate undergoes rapid and vigorous gelation, whereby the polymer chains form an "egg-box" structure. A disadvantage of alginate includes its rheological properties, which produces end results of poor printability and pattern fidelity (Wu et al., 2017).

The use of CNCs confers an array of advantages for TE, such as increased mechanical strength, low density, renewability, and low cytotoxicity. Incorporation of CNC in hydrogel matrices proves advantageous, as $\mathrm{CNC}$ is known to increase the mechanical strength and to induce shear thinning behavior. Wu et al. (2017) demonstrated that a production of a hybrid hydrogel, combining both alginate and CNCs, combines the advantages of the components, and an elimination of the disadvantages is seen. Wu et al. (2017) demonstrated that with an increasing concentration of CNCs, the geometries become significantly well-defined. Wu et al. (2017) also concluded that a 20:40 alginate-to-CNC ratio is the most suitable candidate for tissue printing.

$\mathrm{Wu}$ et al. demonstrated that upon incorporation of fibroblasts and hepatoma cells as a component of the bioink, bioprinting of cell-laden hydrogel-based bioink produces no observable cellular damage, and therefore it can be concluded that the process is cell-compatible. Gelatin allows for fibroblast and hepatoma attachment, increasing cell viability, and does not alter the viscosity and printability of the hydrogel-based bioink (Wu et al., 2017).

\section{THREE-DIMENSIONAL BIOPRINTING OF VOLUMETRIC TISSUES AND ORGANS EMPLOYING HYDROGEL-BASED BIOINKS}

One of the prevalent challenges that cripple the science behind $3 \mathrm{D}$ bioprinting is the development of a bioink that maintains a low viscosity, which allows for effective and efficient printing through a defined nozzle diameter but simultaneously bears a viscosity that allows for the formation of a solid or semisolid structure postprinting. This intermediate viscosity is demonstrating significant challenges impeding the suitability and feasibility for large-scale manufacturing (Kilian et al., 2017). Discussed below are possible solutions to circumvent the aforementioned challenges and enable the fabrication of volumetric tissues and organs through $3 \mathrm{D}$ bioprinting.

\section{Technical Solutions}

Different mammalian cells display an array of highly selective and differential structural morphology; therefore, specific parameters need to be satisfied for the ability to generate physiological relevant structures through the process of $3 \mathrm{D}$ bioprinting (Pati et al., 2016), and the accuracy and printing of tissue mimetics are critical. Current $3 \mathrm{D}$ bioprinting involves the printing of bioinks, from an air medium onto a solid, flat surface (Kilian et al., 2017). As demonstrated by Blaeser et al. (2013), how printing into a medium that consists of a highbuoyant liquid determines accuracy and size of individual hydrogel droplets versus printing in a medium containing air (Blaeser et al., 2013). Figure 3 demonstrates the stepwise process undertaken by Blaeser et al. (2013).

In summary, the aforementioned investigations proved submerged printing, in high-density perfluorocarbon (PFC) fluid, to be a far more superior method when compared to the commonly used method of printing from a medium of air. The hydrogel droplets printed submerged in PFC exhibited droplets with an increased contact angle, decreased the flatness, and decreased diameter as compared to printing in an air medium. These factors collectively improve the spatial resolution of the printer and the production of high-aspect-ratio structures more readily (Blaeser et al., 2013). 


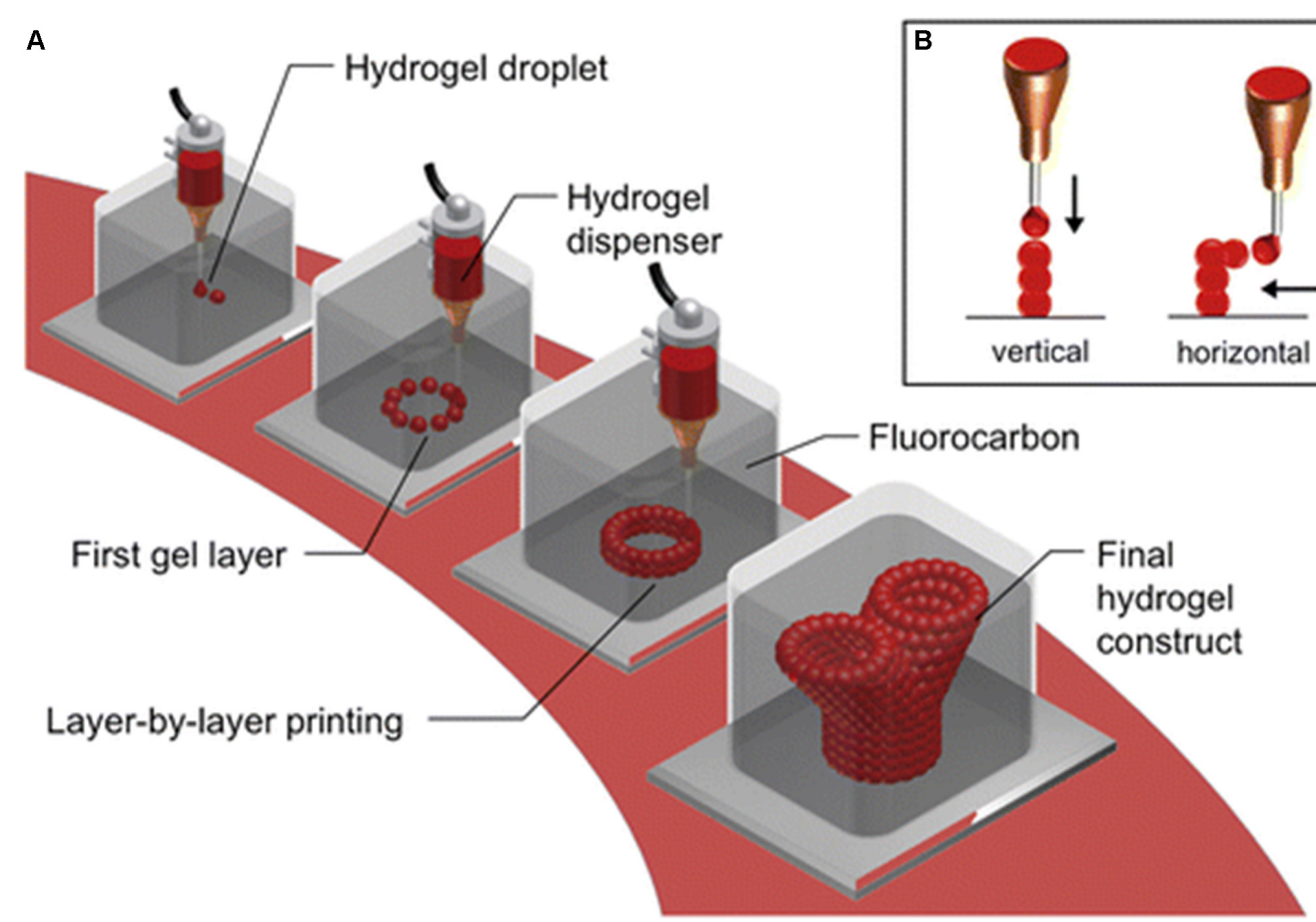

FIGURE 3 | Submerged bioprinting. (A) Bioprinting cell-laden hydrogels (single drops), layer by layer as per a predefined and constructed model. The bioprinting is conducted, by submerging the nozzle, in high-density perfluorocarbons, which are chemical inert and immiscible in both water and oil. Perfluorocarbons are also known to display excellent oxygen and carbon dioxide transport capability, which provides an ideal environment for submerged cells. (B) The hydrogel drops can be printed either in a vertical or lateral dimension. With perfluorocarbon providing a buoyant support, printing can be conducted to produce branching structures, without solid support (Blaeser et al., 2013, Creative Commons license).

\section{Crosslinking Approaches Employed in Synthesis of Hydrogel-Based Bioinks}

To ensure that stability optimization during the process of bioprinting is maintained, the process of crosslinking needs to be considered. As the hydrogel-based bioink is ejected from the nozzle, ideally the hydrogel-based bioink should be subjected to crosslinking (Kilian et al., 2017).

Ahn et al. (2012) demonstrated the instantaneous contact between the printed bioink and a crosslinking agent, with the technique of introducing a crosslinking agent, calcium chloride $\left(\mathrm{CaCl}_{2}\right)$, by aerosol spray. This innovative technique allows for the control of surface gelation during the printing process (Ahn et al., 2012). Application of this technique does bear advantages such as the introduction of a $10 \% \mathrm{wt} / \mathrm{vol}$ of $\mathrm{CaCl}_{2}$ via aerosol dispensing and shows an enhancement in the viscosity of an alginate solution used as a hydrogel-based bioink. The resultant enhanced viscosity of the hydrogel alginate as a bioink significantly improves its inherent mechanical strength conferring the ability to support cellular activities (Jenkins, 2007; Ahn et al., 2012).

Hydrogels that are suitable for photocrosslinking serve to provide attractive biomaterials as they can rapidly undergo crosslinking that can allow for cell compatibility and ensure spatiotemporal control over the gelation process. This process introduces a photoreactive chemical group into the biomaterial to allow for covalent crosslinking (Pereira and Bártolo, 2015).
Photopolymerization can be conducted during the printing process or immediately following dispensing of the biomaterial, to allow for rapid polymer crosslinking between polymer chains (Cui et al., 2012; Billiet et al., 2014).

Free radical polymerization is the most commonly used method for photocrosslinking for hydrogels. Free radical polymerization is a multistep process; as the process proceeds, the number of crosslinks formed increased producing a highly polymerized network via a "chain-growth mechanism" (Pereira and Bártolo, 2015). The disadvantage of this application includes oxygen inhibition, lack of control over the rate of polymerization, and potential generation of heterogeneities within the hydrogel (Lin et al., 2015).

The major concern with photocrosslinking includes the exposure of ultraviolet (UV) light radiation to biological entities. This exposure can generate cytotoxic free radicals and stimulate local inflammation. The addition of UV-A light has demonstrated to reduce the strain placed on biological entities (Gupta et al., 2013). Cellular damage attributed to UV radiation exposure can be limited by selecting a desired wavelength, exposure time, and intensity (Hao et al., 2014).

\section{Modification of Hydrogel-Based Bioinks for Internal Stabilization}

Internal stabilization aids the process of bioprinting volumetric structures. Fundamentally, internal stabilization to improve the 
process of volumetric structure production is to modify the viscosity of the hydrogel-based bioink (Kilian et al., 2017). Considering the science of rheology, bioprinting using nozzlebased technology, following ejection of the hydrogel, the material needs to undergo rapid solidification and structure preservation. However, the alteration of the viscosity to ensure rapid solidification can limit cell encapsulation (Jungst et al., 2016). Promising approaches have been developed to enhance the viscosity of cell-laden hydrogels, while enhancing the shape and fidelity of the fabricated structure (Kilian et al., 2017).

Markstedt et al. (2015) demonstrated that the use of an alginate hydrogel as a bioink bears zero shear viscosity and produces poor shape fidelity during the printing process compared to a bioink consisting of both alginate and nanofibrillated cellulose (NFC), which inherently displays excellent mechanical and biological properties, proved shear thinning, and produced high viscosity and increased printing resolution. This study further demonstrates that the use of an 80:20 alginate and NFC bioink provides $86 \%$ cell survival rate post printing. Therefore, upon the addition of NFC, a novel solution is demonstrated for both biological and mechanical printing limitations (Markstedt et al., 2015).

\section{Provision of Hydrogel-Based Bioink Support for External Stabilization}

External stabilization is a technique that employs the incorporation of a minimum of two materials: a robust and tough material for structural support and a soft material that enables cellular growth and proliferation. This process involves the printing of the two aforementioned materials on separate cartridges (Kilian et al., 2017). This technique provides mechanical support for the soft and cell-laden hydrogel.

Bioprinted human tissue indicated for regenerative TE is essentially formulated to bear the inherent biomechanical properties while mirroring the structural and functional properties of pathologically absent tissue. Schuurman et al. (2011) demonstrate a viable solution that allows for musculoskeletal tissue bioprinting that meets the required goal. This study involves the printing of a hybrid structure, by alternating deposition of thermoplastic fibers and a cell-laden hydrogel. The resultant hybrid hydrogel possesses excellent mechanical properties comparable to the originator tissue. This approach is advantageous as it is not only limited to TE but can also be applied in the field of drug or bioactive substances delivery (Schuurman et al., 2011).

\section{THREE-DIMENSIONAL BIOPRINTING OF MULTIMATERIALS AND MULTICELLULAR COMPONENTS}

The physicochemical properties of the biomaterial confer a critical and restrictive parameter for the 3D bioprinting process. The current technology employed executes the process of $3 \mathrm{D}$ bioprinting by a layer-by-layer process, whereby a layer of biomaterial is printed onto a solid surface, which then serves as a base for a subsequent layer. Based on this technique, the lower layers should bear mechanical strength sufficient to support the subsequent layers to prevent structural collapse. The dispensing of a layer is defined as the "transfer of mechanical, thermal, chemical, and electromagnetic energy, from the bioprinter to cellladen biomaterials or bioink." This transfer of energy may result in the deformation and destruction of cell-laden bioinks, which can occur via membrane permeability alteration and changes in osmotic pressure between the external environment and internal cellular environment (De Maria et al., 2017).

Multiple biological reactions, in vivo, occur at interfaces or surfaces; therefore, a critical parameter that influences the activity and efficacy of a 3D bioprinted hydrogel implant is its microsurface and nanosurface characteristics (Salmasi et al., 2015). The nanotopography of a medical implant is known to induce tissue-specific function and stimulate cell differentiation and growth. Materials that confer nanotopographic properties bear similar properties to growth factors. This attractive property allows for the application of 3D bioprinted hydrogel implant clinically, to induce biological functions (Unadkat et al., 2011; Kim et al., 2013).

Andersen et al. (2010) demonstrated the incorporation of nanoparticles containing small interfering RNA (siRNA) into a poly- $\varepsilon$-caprolactone (PCL) hydrogel scaffold, to ensure spatially controlled development of cells for engineered morphogenesis. Andersen et al. (2010) presented the use of a novel technology that comprised a nanoparticle-based PCL hydrogel scaffold that bears the ability to deliver siRNAs into the cells of the scaffold from a nanoparticle-based implant coating. This technology has demonstrated the ability to modulate the differentiation of stem cells. The incorporation of siRNA onto the seeded cells stimulated a series of gene silencing, resulting in the enhancement of the osteogenic and adipogenic differentiation of mesenchymal stem cell (Andersen et al., 2010).

Another consideration that can be explored is the construction of a self-assembly scaffold. Self-assembling scaffolds refer to scaffold that bears the ability to form non-covalent interactions spontaneously between hydrophilic and hydrophobic amino acid motifs (Kang et al., 2014; Do et al., 2015). This non-covalent selfassembly peptide can be applied for the preparation of functional hydrogels for the purposes of TE, drug delivery, and wound healing. Self-assembly induces peptide-based fibrils to form entangled networks, which reduce solvent flow and stimulate hydrogel formation. Stimulus responsive peptides present an attractive feature, whereby the rate of hydrogelation can be controlled as a function of the environment. An array of peptides has been formulated to allow for the stimulation of self-assembly peptides, which include $\mathrm{pH}$, temperature, light, and enzymatic manipulation (Bowerman and Nilsson, 2010).

The construction of self-assembly peptide hydrogels extends between two material classes, as mentioned previously. These self-assembling hydrogels consist of natural monomers, but do not occur naturally. The utilization of natural amino acids in predetermined sequences can pave the way toward safe and biocompatible biomaterials with the decreased risk of immunogenicity. Alterations of amino acid side chains provide us the ability to alter the physicochemical 
properties of a hydrogel, such as, pore size, fiber diameter, biomechanical properties, sensitivity toward $\mathrm{pH}$, and ionic interactions. The relevance of the incorporation of amino acids allows the conferment of biological properties to a hydrogel (Maude et al., 2013).

Holmes et al. (2000) demonstrated the formulation of a self-assembly peptide scaffold. Under normal physiological conditions, this scaffold exhibited the self-assembling ability, which results in the formation of spontaneous ionic selfcomplementary $\beta$-sheet oligopeptides, which produced a hydrogel, appropriate for neuronal TE. This scaffold displays an attractive property that allows for neuronal cell attachment and promotes neuronal cell proliferation. This study further demonstrated apart from the promising self-assembly capability, the excellent biodegradability, lack of an immune response, and inflammation, upon in vivo implantation. This study provides the platform for the investigation of the potential of this peptidebased bioink to 3D bioprinting. Holmes et al. (2000) provided promising data enabling the use of the investigated hydrogel in the field of TE (Holmes et al., 2000).

The inclusion of a biomaterial or scaffold independent bioprinting techniques for the bioprinting of multicellular and multimaterial structures is a promising consideration that requires attention. Apart from the numerous advantages associated with scaffold bioprinting, there are several drawbacks that impede the TE field, which include cell encapsulation as a result of a biomaterial scaffold, limiting intercellular communication; the biomaterial scaffold commonly decreases cell viability; and method-specific bioprinting resulting in the generation of a biomaterial scaffold with secondary cytotoxic byproduct, which not only impedes viability but may stimulate adverse immune response upon in vivo implantation (Nair et al., 2009; Murphy et al., 2013; Murphy and Atala, 2014; Moldovan, 2018).

Tseng et al. (2013) demonstrated the application of magnetic levitation, for the purpose of lung TE. This technique provides a promising approach to the assembly of biologically organized structures. Magnetic levitation incorporates the use poly-Llysine, magnetic iron oxide $\left(\mathrm{Fe}_{3} \mathrm{O}_{4}\right.$, magnetite), and gold nanoparticles, which allows for particle self-assembly based on electrostatic interactions. Introduction of these nanoparticles to cells induces a cell-magnetic behavior, allowing for magnetic guidance. Magnetic manipulation directs cells to levitate to an air-liquid interface on a culture dish, allowing for cells, to interact, assemble, and proliferate, with the requirement of a temporary scaffold to provide an ECM (Tseng et al., 2013).

\section{STATE-OF-THE-ART NEXT-GENERATION SMART HYDROGELS EMPLOYED FOR 4D BIOPRINTING}

Four-dimensional (4D) bioprinting is a next-generation printing technique that employs the smart (stimuli-responsive) hydrogels for the fabrication of $3 \mathrm{D}$ cell-laden structures. $4 \mathrm{D}$ bioprinting is an extension to the development of $3 \mathrm{D}$ bioprinting, where time is the fourth dimension. The shape or any other desired property evolves under the influence of external stimuli (e.g., $\mathrm{pH}$, temperature; magnetic attraction; oxidation and reduction ability; functional molecules such as enzymes, glucose, or a combination of them) as time progresses. $4 \mathrm{D}$ bioprinting is an attractive technique that enables the fabrication of dynamic ("living") structures with the ability to transform and respond to the external environment over time (Miao et al., 2017).

\section{Applications and Consideration for Smart Hydrogels Employed for Tissue Regeneration}

Natural tissues are heterogeneous structures with complex geometries that are a fundamental requirement for the diverse functions performed. The incorporation of smart hydrogel materials as cell carriers provides a realistic approach to produce multifunctional tissue constructs with dynamic arrangement of native organs and tissues (Yang et al., 2019). The topological nature of tissues and organs is dependent on the cellular microarrangement and nanoarrangement patterns. While 3D bioprinting allows for the fabrication of tissue constructs that mimic the cellular microarrangement and nanoarrangement patterns, the process remains a challenge. Aligned topological cues are an essential parameter for biomedical applications as many native tissue activities such as nerves, muscles, and tendons are significantly affected by its topology (Villar et al., 2011). Kim et al. (2018) synthesized a 3D bioprinted micropatterned PCL microfibril structure. This technique employed the microfibrillation of poly(vinyl)alcohol (PVA) from a PCL/PVA composite simulating the in vitro alignment of skeletal muscle and fusion. The biocompatibility of the microfibril structure type 1 collagen was added as a supplementary hydrogel polymer. Myoblast cellular proliferation and differentiation substantiated the potential use of this microfibrillated structure to imitate structural geometry tissue regeneration (Kim et al., 2018).

The ability to reproduce multifunctional cell-laden hydrogel mimetic scaffolds for biomedical applications is often difficult; however, it is the ultimate objective in tissue regeneration. Recently, the use of smart hydrogels with the ability to replicate the native functionality of the originator cells has received great interest over the past few years. Deng et al. (2016) developed a PCL electroactive shape memory copolymer that demonstrated good elasticity, biodegradability, and an adjustable recovery temperature and able to stimulate myoblast differentiation and proliferation, myotube formation, and myoblast differentiation gene expression (Deng et al., 2016). Zhao et al. (2017b) fabricated a dopamine (DA) bioactive electroactive shape memory polyurethane elastomer. This elastomer comprised a poly(citric acid-co-PCL) (CA-PCL) polyurethane elastomer, bioactive DA, and electroactive aniline hexamer. The elastomer demonstrated excellent deformation shape recovery under physiological conditions. The bioactive and electroactive properties of the elastomer conferred excellent cellular proliferation and viability and mild immunological response following subcutaneous implantation (Zhao et al., 2017b). The results of these studies substantiate the potential 
TABLE 3 | Applications of 4D bioprinted hydrogel structures for tissue regeneration.

\begin{tabular}{|c|c|c|c|c|}
\hline Cellular origin & Cell type & Hydrogel & Stimuli-responsive behavior & References \\
\hline Stem cells & $\begin{array}{l}\text { Mesenchymal stem } \\
\text { cells }\end{array}$ & Polycaprolactone (PCL) & $\begin{array}{l}\text { The fabricated 3D structure demonstrates shape reform ability } \\
\text { within the glass transition temperature range }\left(-8 \text { to } 35^{\circ} \mathrm{C}\right)\end{array}$ & Miao et al., 2016 \\
\hline Bone and cartilage & $\begin{array}{l}\text { Bone marrow-derived } \\
\text { cells and chondrocytes }\end{array}$ & $\begin{array}{l}\text { Dextran grafted } \\
\text { ureido-pyrimidinone }\end{array}$ & $\begin{array}{l}\text { This hydrogel demonstrates rapid solidification } \\
\text { postadministration and self-integration capacity }\end{array}$ & Hou et al., 2015 \\
\hline \multirow[t]{2}{*}{ Muscle } & C2C12 myoblasts & PLC, PVA & $\begin{array}{l}\text { The copolymeric hydrogel of PVA and PCL forms aligned } \\
\text { topological cues when dissolved in water }\end{array}$ & Kim et al., 2018 \\
\hline & C2C12 myoblasts & $\begin{array}{l}\text { Collagen type I and } \\
\text { PVA }\end{array}$ & $\begin{array}{l}\text { This copolymeric hydrogel forms aligned topological cues when } \\
\text { dissolved in water }\end{array}$ & Kim et al., 2017 \\
\hline
\end{tabular}
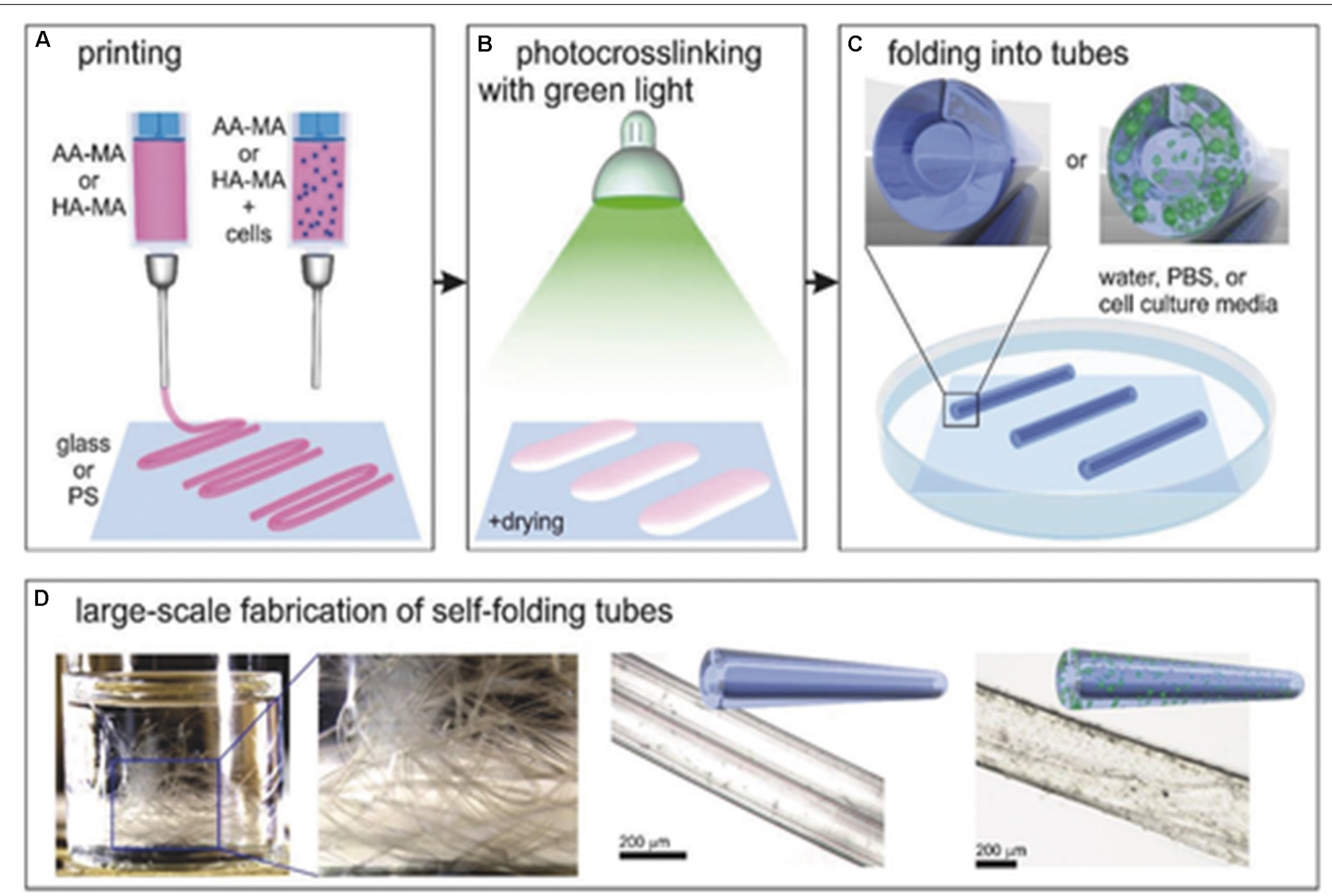

FIGURE 4 | Diagrammatic representation of the 4D bioprinted of a cell-laden self-folding hydrogel scaffold. (A) Bioprinting of methacrylated alginate (AA-MA) and methacrylated hyaluronic acid (HA-MA) with and without the incorporation of cells. (B) Photocrosslinking the hydrogel in the presence of green (530 nm) light with mild drying. (C) The self-folding of hydrogels in an aqueous environment. (D) Examples of the self-folding hydrogels. Reproduced with permission from Kirillova et al. (2017).

use of hydrogel with shape morphing properties to replicate physiological functions to induce favorable and desired cellular behavior.

Biomaterials such as hydrogels, employed for the fabrication of tissue constructs for tissue regenerations, should be appropriate and have comparable mechanical strength to the native tissues and organs. Inappropriate mechanical strength can result in the detachment of an implant or tissue ingrowth (Wieding et al., 2012). Antony et al. (2018) designed a hydroxyapatite scaffold grafted with polyethylene glycol methacrylate. The grafted polymeric monomer contributed to an enhanced interfacial interaction between colloidal particles of the scaffold and improved mechanical strength of the shape memory polymeric scaffold and excellent cell viability (Antony et al., 2018).

\section{Biomedical Applications of 4D Bioprinted Smart Hydrogels for Tissue Regeneration}

Recently, the application and use of $4 \mathrm{D}$ bioprinted smart (stimuliresponsive) hydrogels for tissue regeneration have received 
significant attention. The fabrication of $4 \mathrm{D}$ bioprinted tissue constructs is defined as the process of 3D bioprinting stimuliresponsive (smart) hydrogels as tissue scaffolds for in vivo and in vitro tissue regeneration (Ong et al., 2018). Table 3 outlines the application and successes of $4 \mathrm{D}$ bioprinted hydrogel cellladen scaffolds.

Hendrikson et al. (2017) developed a 4D bioprinted cell-laden scaffold for tissue regeneration. The use of a shape memory polyurethane was employed as the primary polymer to cellular alignment and stretching. This study exploited the transition temperature of polyurethane $\left(34^{\circ} \mathrm{C}\right)$ to allow for cell stabilization and prior to shape recovery process at $37^{\circ} \mathrm{C}$. This study demonstrated excellent cellular viability and proliferation through shape recovery (Hendrikson et al., 2017).

Jamal et al. (2013) fabricated a bio-origami cell-laden hydrogel scaffold with the ability to undergo self-folding. The photopatterned poly(ethylene glycol) (PEG)-based hydrogel bilayers were able to self-assemble into anatomically appropriate micrometer scale geometries. The self-folding ability was attributed to the swelling properties of the PEG bilayers in an aqueous environment (Jamal et al., 2013). Kirillova et al. (2017) also fabricated a self-folding cell-laden hydrogel; however, the ability to undergo self-folding was related to the degree of crosslinking rather than the swelling ratio (Kirillova et al., 2017). Figure 4 illustrates the self-folding effect of the cell-laden hydrogel scaffold.

\section{FUTURE OUTLOOK AND CONCLUDING REMARKS}

The selection of materials employing 3D bioprinting is significantly dependent on the biocompatibility with cellular growth and proliferation and its printing ability such as viscosity, extrudability, and mechanical stability. Therefore, the successes for the fabrication of tissue constructs are considerably limited to alginate, cellulose, gelatin, polyacrylates, and hyaluronic acid. The fundamental understanding and integration of cellular microenvironment with material science are needed for the development of a novel bioink for the fabrication of 3D bioprinted tissue constructs. The development of a functionally responsive material that responds to an external stimulus, inducing transitional changes within the cellladen matrix, may prove useful in circumventing challenges associated with 3D bioprinting hydrogel-based structures. While 4D bioprinting serves to provide a solution for the above challenges, human-scale fabrication of tissue constructs demonstrates limitations.

Human organs consist of an array of different cell species and hence different cellular matrix. The current limitation for 3D bioprinting human organs for transplantation is the use of a single extrusion nozzle and the use of one to three cell species, and the degree to which the resultant $3 \mathrm{D}$ structure produces the complexity of a natural human organ is largely unclear (Feinberg and Miller, 2017). Kang et al. (2016) demonstrate a novel system that dispenses cell-laden hydrogels and biodegradable polymers, as to overcome previous $3 \mathrm{D}$ bioprinting limitations such as the vascularization of 3D bioprinted constructs, integrity, strength, and size. This was carried out by employing multidispensing nozzles for the dispensing of multiple cell species and polymers in an isolated constructed model (Kang et al., 2016).

Other limitations include that the choice of hydrogel employed for the fabrication of 3D tissue constructs should be biocompatible and support cellular viability. To fabricate multifunctional structures of appropriate mechanical strength to support cellular viability and printability remains a challenge. The crosslinking agents employed to stabilize the final 3D tissue construct should be non-cytotoxic. Current applications of 3D and 4D bioprinted cell-laden hydrogels have not met these requirements entirely. The optimization of biopolymers used in the fabrication of hybrid structures can circumvent these challenges. Furthermore, multifunctional tissues and organs are subjected to the multiple simultaneous stimuli; therefore, fabricated 3D structures consist of multiple smart hydrogels. Therefore, the need for highresolution bioprinting is significantly required for the precise placement of multiple smart hydrogels in a specific area (Yang et al., 2019).

Over the next few decades, 3D bioprinting serves as one of the primary focal points of TE. While research scientists across the field of TE determine the minimum conditions for organlevel function reconstitution in vitro for in vivo application, a multidisciplinary approach is required that consists of practicing clinical surgeons and pharmacists that is not just limited to research scientists. The development of this multidisciplinary healthcare team serves to provide the TE field, with critical parameters that connect engineered structures to the human recipient (Feinberg and Miller, 2017).

\section{AUTHOR CONTRIBUTIONS}

PR compiled this review manuscript and is the lead author of this manuscript. LT provided content and editorial input based on expertise in stimulus-responsive biopolymers. YC provided content and editorial input based on expertise in 3D printing. PK undertook initial review and provided editorial input. VP provided content input based on extensive expertise in biopolymers and is the corresponding author. VP, YC, LT, and PK co-conceptualized the research topic of PR on which this review is based.

\section{FUNDING}

This work was supported by the National Research Foundation (NRF) of South Africa and the University of the Witwatersrand, Johannesburg. 


\section{REFERENCES}

Ahn, S., Lee, H., Bonassar, L. J., and Kim, G. (2012). Cells (MC3T3-E1)-laden alginate scaffolds fabricated by a modified solid-freeform fabrication process supplemented with an aerosol spraying. Biomacromolecules 13, 2997-3003. doi: $10.1021 / \mathrm{bm} 3011352$

Andersen, M. Ø, Nygaard, J. V., Burns, J. S., Raarup, M. K., Nyengaard, J. R., Bünger, C., et al. (2010). siRNA nanoparticle functionalization of nanostructured scaffolds enables controlled multilineage differentiation of stem cells. Mol. Ther. 18, 2018-2027. doi: 10.1038/mt.2010.166

Antony, G. J. M., Aruna, S., and Raja, S. (2018). Enhanced mechanical properties of acrylate based shape memory polymer using grafted hydroxyapatite. J. Polym. Res. 25:120.

Billiet, T., Gevaert, E., De Schryver, T., Cornelissen, M., and Dubruel, P. (2014). The $3 \mathrm{D}$ printing of gelatin methacrylamide cell-laden tissue-engineered constructs with high cell viability. Biomaterials 35, 49-62. doi: 10.1016/j.biomaterials.2013. 09.078

Billiet, T., Vandenhaute, M., Schelfhout, J., Van Vlierberghe, S., and Dubruel, P. (2012). A review of trends and limitations in hydrogel-rapid prototyping for tissue engineering. Biomaterials 33, 6020-6041. doi: 10.1016/j.biomaterials. 2012.04.050

Blaeser, A., Duarte Campos, D. F., Weber, M., Neuss, S., Theek, B., Fischer, H., et al. (2013). Biofabrication under fluorocarbon: a novel freeform fabrication technique to generate high aspect ratio tissue-engineered constructs. Bioresearch Open Access 2, 374-384. doi: 10.1089/biores.2013.0031

Boland, T., Mironov, V., Gutowska, A., Roth, E. A., and Markwald, R. R. (2003). Cell and organ printing 2: fusion of cell aggregates in three-dimensional gels. Anatom. Rec. Part A 272, 497-502.

Bowerman, C. J., and Nilsson, B. L. (2010). A reductive trigger for peptide selfassembly and hydrogelation. J. Am. Chem. Soc. 132, 9526-9527. doi: 10.1021/ ja1025535

Chang, R., Nam, J., and Sun, W. (2008). Effects of dispensing pressure and nozzle diameter on cell survival from solid freeform fabrication-based direct cell writing. Tissue Eng. Part A 14, 41-48. doi: 10.1089/ten.a.2007.0004

Cui, H., Nowicki, M., Fisher, J. P., and Zhang, L. G. (2017). 3D bioprinting for organ regeneration. Adv. Healthc. Mater. 6:1601118.

Cui, X., Boland, T., Dd'lima, D., and Lotz, M. K. (2012). Thermal inkjet printing in tissue engineering and regenerative medicine. Recent Patents Drug Deliv. Formulat. 6, 149-155.

Cui, X., Dean, D., Ruggeri, Z. M., and Boland, T. (2010). Cell damage evaluation of thermal inkjet printed Chinese hamster ovary cells. Biotechnol. Bioeng. 106, 963-969. doi: 10.1002/bit.22762

De Maria, C., Vozzi, G., and Moroni, L. (2017). Multimaterial, heterogeneous, and multicellular three-dimensional bioprinting. MRS Bull. 42, 578-584.

Deng, Z., Guo, Y., Zhao, X., Li, L., Dong, R., Guo, B., et al. (2016). Stretchable degradable and electroactive shape memory copolymers with tunable recovery temperature enhance myogenic differentiation. Acta Biomater. 46, 234-244. doi: 10.1016/j.actbio.2016.09.019

Derby, B. (2012). Printing and prototyping of tissues and scaffolds. Science 338, 921-926. doi: 10.1126/science. 1226340

Do, A. V., Khorsand, B., Geary, S. M., and Salem, A. K. (2015). 3D printing of scaffolds for tissue regeneration applications. Adv. Healthc. Mater. 4, 17421762. doi: 10.1002/adhm.201500168

Duan, B., Hockaday, L. A., Kang, K. H., and Butcher, J. T. (2013). 3D bioprinting of heterogeneous aortic valve conduits with alginate/gelatin hydrogels. J. Biomed. Mater. Res. Part A 101, 1255-1264. doi: 10.1002/jbm.a.34420

Fedorovich, N. E., De Wijn, J. R., Verbout, A. J., Alblas, J., and Dhert, W. J. (2008). Three-dimensional fiber deposition of cell-laden, viable, patterned constructs for bone tissue printing. Tissue Eng. Part A 14, 127-133. doi: 10.1089/ten.a.2007. 0158

Feinberg, A. W., and Miller, J. S. (2017). Progress in three-dimensional bioprinting. MRS Bull. 42, 557-562. doi: 10.1557/mrs.2017.166

Gaetani, R., Doevendans, P. A., Metz, C. H., Alblas, J., Messina, E., Giacomello, A., et al. (2012). Cardiac tissue engineering using tissue printing technology and human cardiac progenitor cells. Biomaterials 33, 1782-1790. doi: 10.1016/ j.biomaterials.2011.11.003

Griffith, L. G., and Swartz, M. A. (2006). Capturing complex 3D tissue physiology in vitro. Nat. Rev. Mol. Cell Biol. 7:211.
Guillemot, F., Mironov, V., and Nakamura, M. (2010a). Bioprinting is coming of age: report from the International Conference on Bioprinting and Biofabrication in Bordeaux (3B'09). Biofabrication 2:010201. doi: 10.1088/1758$5082 / 2 / 1 / 010201$

Guillemot, F., Souquet, A., Catros, S., and Guillotin, B. (2010b). Laser-assisted cell printing: principle, physical parameters versus cell fate and perspectives in tissue engineering. Nanomedicine 5, 507-515. doi: 10.2217/nnm.10.14

Gupta, A., Avci, P., Dai, T., Huang, Y.-Y., and Hamblin, M. R. (2013). Ultraviolet radiation in wound care: sterilization and stimulation. Adv. Wound Care 2, 422-437.

Gupta, S. K., Saxena, P., Pant, V. A., and Pant, A. B. (2012). Release and toxicity of dental resin composite. Toxicology international 19, 225. doi: 10.4103/09716580.103652

Hao, Y., Shih, H., Muòoz, Z., Kemp, A., and Lin, C.-C. (2014). Visible light cured thiol-vinyl hydrogels with tunable degradation for 3D cell culture. Acta Biomater. 10, 104-114. doi: 10.1016/j.actbio.2013.08.044

Hendrikson, W. J., Rouwkema, J., Clementi, F., Van Blitterswijk, C. A., Farè, S., and Moroni, L. (2017). Towards 4D printed scaffolds for tissue engineering: exploiting 3D shape memory polymers to deliver time-controlled stimulus on cultured cells. Biofabrication 9:031001. doi: 10.1088/1758-5090/aa8114

Hoffman, A. S. (2012). Hydrogels for biomedical applications. Adv. Drug Deliv. Rev. 64, 18-23.

Holmes, T. C., De Lacalle, S., Su, X., Liu, G., Rich, A., and Zhang, S. (2000). Extensive neurite outgrowth and active synapse formation on self-assembling peptide scaffolds. Proc. Natl. Acad. Sci. U.S.A. 97, 6728-6733. doi: 10.1073/pnas. 97.12.6728

Hou, S., Wang, X., Park, S., Jin, X., and Ma, P. X. (2015). Rapid self-integrating, injectable hydrogel for tissue complex regeneration. Adv. Healthc. Mater. 4, 1491-1495. doi: 10.1002/adhm.201500093

Ilkhanizadeh, S., Teixeira, A. I., and Hermanson, O. (2007). Inkjet printing of macromolecules on hydrogels to steer neural stem cell differentiation. Biomaterials 28, 3936-3943.

Jamal, M., Kadam, S. S., Xiao, R., Jivan, F., Onn, T. M., Fernandes, R., et al. (2013). Bio-origami hydrogel scaffolds composed of photocrosslinked PEG bilayers. Adv. Healthc. Mater. 2, 1142-1150. doi: 10.1002/adhm.201200458

Jenkins, M. (2007). Biomedical Polymers. Amsterdem: Elsevier.

Jungst, T., Smolan, W., Schacht, K., Scheibel, T., and Groll, J. (2016). Strategies and molecular design criteria for 3D printable hydrogels. Chem. Rev. 116, 1496-1539.

Kamata, H., Li, X., Chung, U.-I., and Sakai, T. (2015). Design of hydrogels for biomedical applications. Adv. Healthc. Mater. 4, 2360-2374. doi: 10.1002/adhm. 201500076

Kang, M. K., Colombo, J. S., D’souza, R. N., and Hartgerink, J. D. (2014). Sequence effects of self-assembling multidomain peptide hydrogels on encapsulated SHED cells. Biomacromolecules 15, 2004-2011. doi: 10.1021/bm500075r

Kang, H. W., Lee, S. J., Ko, I. K., Kengla, C., Yoo, J. J., and Atala, A. (2016). A $3 \mathrm{D}$ bioprinting system to produce human-scale tissue constructs with structural integrity. Nat. Biotechnol. 34:312. doi: 10.1038/nbt.3413

Kilian, D., Ahlfeld, T., Akkineni, A. R., Lode, A., and Gelinsky, M. (2017). Three-dimensional bioprinting of volumetric tissues and organs. MRS Bull. 42, 585-592. doi: 10.1016/j.actbio.2019.01.009

Kim, H. N., Jiao, A., Hwang, N. S., Kim, M. S., Kim, D.-H., and Suh, K.-Y. (2013). Nanotopography-guided tissue engineering and regenerative medicine. $A d v$. Drug Deliv. Rev 65, 536-558. doi: 10.1016/j.addr.2012.07.014

Kim, M., Kim, W., and Kim, G. (2017). Topologically micropatterned collagen and poly ( $\varepsilon$-caprolactone) struts fabricated using the poly (vinyl alcohol) fibrillation/leaching process to develop efficiently engineered skeletal muscle tissue. ACS Appl. Mater. Interf. 9, 43459-43469. doi: 10.1021/acsami. $7 \mathrm{~b} 14192$

Kim, W., Kim, M., and Kim, G. H. (2018). 3D-printed biomimetic scaffold simulating microfibril muscle structure. Adv. Funct. Mater. 28:1800405.

Kirillova, A., Maxson, R., Stoychev, G., Gomillion, C. T., and Ionov, L. (2017). 4D biofabrication using shape-morphing hydrogels. Adv. Mater. 29:1703443.

Langer, R., and Vacanti, J. P. (1993). Tissue engineering. Science 260:920.

Lee, J. W., Kim, J. Y., and Cho, D.-W. (2010). Solid free-form fabrication technology and its application to bone tissue engineering. Int. J. Stem Cells 3:85.

Lee, W., Debasitis, J. C., Lee, V. K., Lee, J.-H., Fischer, K., Edminster, K., et al. (2009). Multi-layered culture of human skin fibroblasts and keratinocytes 
through three-dimensional freeform fabrication. Biomaterials 30, 1587-1595. doi: $10.1016 /$ j.biomaterials.2008.12.009

Lin, C. C., Ki, C. S., and Shih, H. (2015). Thiol-norbornene photoclick hydrogels for tissue engineering applications. J. Appl. Polym. Sci. 132:41563.

Mandrycky, C., Wang, Z., Kim, K., and Kim, D.-H. (2016). 3D bioprinting for engineering complex tissues. Biotechnol. Adv. 34, 422-434. doi: 10.1016/j. biotechadv.2015.12.011

Marchioli, G., van Gurp, L., Van Krieken, P. P., Stamatialis, D., Engelse, M., Van Blitterswijk, C. A., et al. (2015). Fabrication of three-dimensional bioplotted hydrogel scaffolds for islets of Langerhans transplantation. Biofabrication 7:025009. doi: 10.1088/1758-5090/7/2/025009

Markstedt, K., Mantas, A., Tournier, I., Martínez Ávila, C., Hägg, D., and Gatenholm, P. (2015). 3D bioprinting human chondrocytes with nanocellulose-alginate bioink for cartilage tissue engineering applications. Biomacromolecules 16, 1489-1496. doi: 10.1021/acs.biomac.5b00188

Maude, S., Ingham, E., and Aggeli, A. (2013). Biomimetic self-assembling peptides as scaffolds for soft tissue engineering. Nanomedicine 8, 823-847. doi: 10.2217/ nnm. 13.65

Miao, S., Castro, N., Nowicki, M., Xia, L., Cui, H., Zhou, X., et al. (2017). 4D printing of polymeric materials for tissue and organ regeneration. Mater. Today 20, 577-591. doi: 10.1016/j.mattod.2017.06.005

Miao, S., Zhu, W., Castro, N. J., Leng, J., and Zhang, L. G. (2016). Four-dimensional printing hierarchy scaffolds with highly biocompatible smart polymers for tissue engineering applications. Tissue Eng. Part C 22, 952-963. doi: 10.1089/ ten.tec.2015.0542

Mironov, V., Reis, N., and Derby, B. (2006). Bioprinting: a beginning. Tissue Eng. $12,631-634$

Moldovan, N. I. (2018). Progress in scaffold-free bioprinting for cardiovascular medicine. J. Cell Mol. Med. 22, 2964-2969. doi: 10.1111/jcmm.13598

Murphy, S. V., and Atala, A. (2014). 3D bioprinting of tissues and organs. Nat. Biotechnol. 32:773.

Murphy, S. V., Skardal, A., and Atala, A. (2013). Evaluation of hydrogels for bioprinting applications. J. Biomed. Mater. Res. Part A 101, 272-284. doi: 10.1002/ jbm.a.34326

Nair, K., Gandhi, M., Khalil, S., Yan, K. C., Marcolongo, M., Barbee, K., et al. (2009). Characterization of cell viability during bioprinting processes. Biotechnol. J. 4, 1168-1177. doi: 10.1002/biot.200900004

Ong, C. S., Nam, L., Ong, K., Krishnan, A., Huang, C. Y., Fukunishi, T., et al. (2018), $3 \mathrm{D}$ and $4 \mathrm{D}$ bioprinting of the myocardium: current approaches, challenges, and future prospects. Biomed Res. Int. 2018:6497242. doi: 10.1155/2018/6497242

Pati, F., Gantelius, J., and Svahn, H. A. (2016). 3D bioprinting of tissue/organ models. Angewandte Chem. Int. Edn. 55, 4650-4665.

Peppas, N. A., and Khademhosseini, A. (2016). Make better, safer biomaterials. Nature News 540, 335.

Pereira, R. F., and Bártolo, P. J. (2015). 3D bioprinting of photocrosslinkable hydrogel constructs. J. Appl. Polym. Sci. 132, 1-15. doi: 10.1088/1758-5090/8/ 2/025019

Ringeisen, B. R., Othon, C. M., Barron, J. A., Young, D., and Spargo, B. J. (2006). Jet-based methods to print living cells. Biotechnol. J. 1, 930-948.

Rutz, A. L., Lewis, P. L., and Shah, R. N. (2017). Toward next-generation bioinks: tuning material properties pre-and post-printing to optimize cell viability. MRS Bull. 42, 563-570.

Salmasi, S., Kalaskar, D. M., Yoon, W.-W., Blunn, G. W., and Seifalian, A. M. (2015). Role of nanotopography in the development of tissue engineered 3D organs and tissues using mesenchymal stem cells. World J. Stem Cells 7:266. doi: $10.4252 /$ wjsc.v7.i2.266

Schuurman, W., Khristov, V., Pot, M. W., Van Weeren, P. R., Dhert, W. J., and Malda, J. (2011). Bioprinting of hybrid tissue constructs with tailorable mechanical properties. Biofabrication 3:021001. doi: 10.1088/1758-5082/3/2/ 021001

Seol, Y.-J., Kang, H.-W., Lee, S. J., Atala, A., and Yoo, J. J. (2014). Bioprinting technology and its applications. Eur. J. Cardiothorac. Surg. 46, 342-348. doi: 10.1093/ejcts/ezu148

Sithole, M. N., Kumar, P., Du Toit, L. C., Marimuthu, T., Choonara, Y. E., and Pillay, V. (2018). A 3D bioprinted in situ conjugated-co-fabricated scaffold for potential bone tissue engineering applications. J. Biomed. Mater. Res. Part A 106, 1311-1321. doi: 10.1002/jbm.a.36333
Stevens, M. M. (2008). Biomaterials for bone tissue engineering. Mater. Today 11, $18-25$.

Tekin, E., Smith, P. J., and Schubert, U. S. (2008). Inkjet printing as a deposition and patterning tool for polymers and inorganic particles. Soft Matter 4, 703-713.

Thomas, D. J. (2016). Could 3D bioprinted tissues offer future hope for microtia treatment? Int. J. Surg. 32, 43-44. doi: 10.1016/j.ijsu.2016.06.036

Tseng, H., Gage, J. A., Raphael, R. M., Moore, R. H., Killian, T. C., GrandeAllen, K. J., et al. (2013). Assembly of a three-dimensional multitype bronchiole coculture model using magnetic levitation. Tissue Eng. Part C 19, 665-675. doi: 10.1089/ten.TEC.2012.0157

Unadkat, H. V., Hulsman, M., Cornelissen, K., Papenburg, B. J., Truckenmüller, R. K., Carpenter, A. E., et al. (2011). An algorithm-based topographical biomaterials library to instruct cell fate. Proc. Natl. Acad. Sci. U.S.A. 108, 16565-16570. doi: 10.1073/pnas.1109861108

Vijayavenkataraman, S., Yan, W.-C., Lu, W. F., Wang, C.-H., and Fuh, J. Y. H. (2018). 3D bioprinting of tissues and organs for regenerative medicine. Adv. Drug Deliv. Rev. 132, 296-332. doi: 10.1016/j.addr.2018. 07.004

Villar, G., Heron, A. J., and Bayley, H. (2011). Formation of droplet networks that function in aqueous environments. Nat. Nanotechnol. 6:803. doi: 10.1038/ nnano.2011.183

Wieding, J., Jonitz, A., and Bader, R. (2012). The effect of structural design on mechanical properties and cellular response of additive manufactured titanium scaffolds. Materials 5, 1336-1347.

Wu, L., Zhu, W.-T., Wang, J., Liu, J., and Zeng, Q.-B. (2017). [Preparation of nanoparticles for sustained insulin release using poly (ethylene glycol) -poly ( $\varepsilon$ caprolactone)-poly (N, N-diethylamino-2-ethylmethaerylate)]. J. S. Med. Univ. $37,975-982$.

Xu, F., Wu, C. A. M., Rengarajan, V., Finley, T. D., Keles, H. O., Sung, Y., et al. (2011). Three-dimensional magnetic assembly of microscale hydrogels. Adv. Mater. 23, 4254-4260. doi: 10.1002/adma.201101962

Yang, G. H., Yeo, M., Koo, Y. W., and Kim, G. H. (2019). 4D Bioprinting: Technological Advances in Biofabrication. Macromol. Biosci. 19:1800441. doi: 10.1002/mabi.201800441

Yao, H., Marcheselli, C., Afanasiev, A., Lähdesmäki, I., and Parviz, B. A. (2012). "A soft hydrogel contact lens with an encapsulated sensor for tear glucose monitoring," in Proceedings of 2012 IEEE 25th International Conference on Micro Electro Mechanical Systems (MEMS) (Paris: IEEE), 769-772.

Zhang, X., and Zhang, Y. (2015). Tissue engineering applications of threedimensional bioprinting. Cell Biochem. Biophys. 72, 777-782. doi: 10.1007/ s12013-015-0531-x

Zhang, Y. S., and Khademhosseini, A. (2017). Advances in engineering hydrogels. Science 356:eaaf3627. doi: 10.1126/science.aaf3627

Zhao, L., Wang, L., Zhang, Y., Xiao, S., Bi, F., Zhao, J., et al. (2017a). Glucose oxidase-based glucose-sensitive drug delivery for diabetes treatment. Polymers 9:255. doi: 10.3390/polym9070255

Zhao, X., Dong, R., Guo, B., and Ma, P. X. (2017b). Dopamine-incorporated dual bioactive electroactive shape memory polyurethane elastomers with physiological shape recovery temperature, high stretchability, and enhanced C2C12 myogenic differentiation. ACS Appl. Mater. Interf. 9, 29595-29611. doi: 10.1021/acsami.7b10583

Zhao, L., Xiao, C., Ding, J., Zhuang, X., Gai, G., Wang, L., et al. (2015). Competitive binding-accelerated insulin release from a polypeptide nanogel for potential therapy of diabetes. Polymer Chem. 6, 3807-3815.

Conflict of Interest: The authors declare that the research was conducted in the absence of any commercial or financial relationships that could be construed as a potential conflict of interest.

Copyright (c) 2020 Ramiah, du Toit, Choonara, Kondiah and Pillay. This is an open-access article distributed under the terms of the Creative Commons Attribution License (CC BY). The use, distribution or reproduction in other forums is permitted, provided the original author(s) and the copyright owner(s) are credited and that the original publication in this journal is cited, in accordance with accepted academic practice. No use, distribution or reproduction is permitted which does not comply with these terms. 